\title{
Characteristic Analysis of Mixed Traffic Flow of Regular and Autonomous Vehicles Using Cellular Automata
}

\author{
Yangzexi Liu, ${ }^{1}$ Jingqiu Guo, ${ }^{1}$ John Taplin, ${ }^{2}$ and Yibing Wang ${ }^{3}$ \\ ${ }^{1}$ Transportation Engineering, Tongji University, Shanghai, China \\ ${ }^{2}$ Business School, The University of Western Australia, Crawley, WA, Australia \\ ${ }^{3}$ College of Civil Engineering \& Architecture, Zhejiang University, Hangzhou, China \\ Correspondence should be addressed to Yibing Wang; wangyibing@zju.edu.cn
}

Received 12 May 2017; Revised 18 July 2017; Accepted 10 August 2017; Published 1 October 2017

Academic Editor: Xiaobo Qu

Copyright (C) 2017 Yangzexi Liu et al. This is an open access article distributed under the Creative Commons Attribution License, which permits unrestricted use, distribution, and reproduction in any medium, provided the original work is properly cited.

\begin{abstract}
The technology of autonomous vehicles is expected to revolutionize the operation of road transport systems. The penetration rate of autonomous vehicles will be low at the early stage of their deployment. It is a challenge to explore the effects of autonomous vehicles and their penetration on heterogeneous traffic flow dynamics. This paper aims to investigate this issue. An improved cellular automaton was employed as the modeling platform for our study. In particular, two sets of rules for lane changing were designed to address mild and aggressive lane changing behavior. With extensive simulation studies, we obtained some promising results. First, the introduction of autonomous vehicles to road traffic could considerably improve traffic flow, particularly the road capacity and free-flow speed. And the level of improvement increases with the penetration rate. Second, the lane-changing frequency between neighboring lanes evolves with traffic density along a fundamental-diagram-like curve. Third, the impacts of autonomous vehicles on the collective traffic flow characteristics are mainly related to their smart maneuvers in lane changing and car following, and it seems that the car-following impact is more pronounced.
\end{abstract}

\section{Introduction}

During the last decade, there has been an intensive effort to develop a variety of vehicle automation systems. Besides their remarkable capabilities in performing various driving tasks, automated/autonomous vehicles may have the potential to influence the operation of traffic systems in terms of efficiency, safety, and greenness [1-5]. More specifically, autonomous vehicles (AVs) have negligible reaction time delays as compared to regular vehicles (RVs) and are able to run with much less spacing or headway with possibly a broad spectrum of speed. It is therefore expected that traffic capacity of roads with AVs would be increased [6-9]. AVs are also able to access the running status of neighboring vehicles so as to make more informed and smarter decisions on their lanechange maneuvers. This could also have a positive impact on road capacity. In addition, AVs may help decrease fuel consumption and emissions [10]. Nevertheless, current vehicle automation technologies are typically developed to benefit individual vehicles, without a clear view of the possible advantages and disadvantages they may contribute to the characteristics of traffic flow.

The penetration rate of autonomous vehicles will be low at the early stage of their deployment, and RVs and AVs are expected to travel together on roads over a long period of time. The mixture of AVs and RVs may reveal some complicated traffic flow characteristics unlike that with only RVs $[11,12]$. So far, studies on such mixed traffic flow are still quite limited [13, 14]. Appropriate models and modeling approaches are needed, at all (microscopic, mesoscopic, and macroscopic) levels, which could allow for a proper reflection of evolving dynamics and capabilities of mixed traffic flow.

This paper intends to explore the characteristics of mixed traffic flow of RVs and AVs, including capacity, fundamental diagram, and lane-changing frequency. Since AVs are not yet in the market, current investigations concerning AVs are performed either via field experiments, which mainly address issues at the individual vehicle level, or via simulation studies 
that could also deliver implications at the traffic flow level. Available simulation studies are not yet sufficient. And (1) effects of AVs were neither fully analyzed nor comprehensively interpreted; (2) identified effects were not always consistent; (3) no unified modeling approach is available. This paper follows the simulation avenue. Since our major concern is about individual vehicles' behavior on overall traffic flow dynamics, we would choose either mesoscopic simulation models like cellular automata (CA) or microscopic simulation models like VISSIM and AIMSUN for this work. As discussed in the literature, an important issue is the balance between the model performance and complexity. Considering the capability of CA in modeling complex nonlinear traffic flow dynamics despite its essential simplicity, we have decided to use CA for this study. More specifically, an improved cellular automata (CA) model is employed in this work. A brief literature review on CA is presented below.

The first cellular automata model that was introduced to traffic simulation is the NaSch model proposed by Nagel and Schreckenberg [15]. CA is essentially a discrete spatiotemporal dynamic system based on local rules [16]. Using CA, complexity and emergence in traffic systems can be modeled and simulated with terse conditions and rules [17]. Because of its simplicity, efficiency, and effectiveness, a large number of extended CA models have been developed following the NaSch model [18]. For instance, Chowdhury et al. [19] developed the Symmetric Two-lane Cellular Automata (STCA) model, which was later expanded by Pederson and Ruhoff [20] to the multilane case. CA models were also used to mimic heterogeneous traffic flow [21-24].

This work models and simulates heterogeneous traffic flow of RVs and AVs using an improved STCA model, in an attempt to identify impacts of AVs on mixed traffic flow dynamics. Different levels of penetration rate of AVs are considered to reflect gradual growth of AVs in traffic flow. At the core of our CA-based mesoscopic traffic simulation model are two specific sets of lane-changing rules for AVs and RVs. One major goal of this research is to determine the AV impact on lane-changing frequency of all involved vehicles. For this reason, a brief literature review on the study of lane changing is presented below.

Frequent lane changes certainly affect traffic flow [25], and improper lane changing has been identified as a main source of traffic congestion and accidents [26, 27]. Gipps introduced probably the first well-known lane-changing model for urban traffic [28], while several other models were developed on the basis of Gipps's model and extended to the freeway case $[15,29]$. Ahmed et al. [30] applied the random utility theory to model lane-changing behavior and defined the lane-changing decision process. Toledo [31] developed a discrete choice framework to model integrated lane changes and estimated the relevant parameters. Under the condition of dense or congested traffic, a vehicle attempting to change lanes needs cooperation from at least one following vehicle in the target lane. Hidas [25] developed a cooperative lanechanging model based on a "driver courtesy" concept. The vehicle that wants to change lane sends a courtesy request to subsequent vehicles in the target lanes; the request is evaluated by each subsequent vehicle. If a vehicle provides courtesy to the requesting vehicle, it reduces its acceleration to ensure that a free gap of sufficient length is created.

By comprehensively reviewing previous works on lane changes, Kesting et al. [32] proposed the model MOBIL (Minimizing Overall Braking Induced by Lane changes) to address cooperative lane changing of intelligent vehicles. MOBIL emulates the lane-changing decision as a trade-off between the incentive that a vehicle intends to do lane changing so as to gain a higher speed and the politeness that this vehicle would exhibit so as to introduce the least disturbance possible to the adjacent vehicles in the target lane. Based on MOBIL, other researchers further studied intelligent lane-changing models [33]. On the empirical side, the studies of lanechanging behavior were far less comprehensive than those of longitudinal driving behavior (such as car following) due to the lack of extensive vehicle trajectory data [34]. The emergence of autonomous and connected vehicle technology offers some great future opportunities.

The paper is organized as follows. Section 2 introduces the modeling and simulation framework based on improved cellular automata and particularly presents two specific sets of rules for lane changing. Section 3 conducts extensive simulation studies, with promising results presented. Section 4 concludes the paper.

\section{Modeling}

2.1. Modeling Setup. A typical traffic CA model accounts for three key components: the road environment, the cells' states, and local transition rules. We consider a three-lane stretch of a freeway, which is represented by a lattice of a number $L$ of cells per lane. The cell length is set to be 5 meters, approximately the average length of a vehicle. The simulation time step is set to be 1 second, and the simulation time horizon is of 10,000 time steps. The speed of any regular vehicle is ane of the six discrete levels, which are $0-5$ cells per time step. " 0 " means that the vehicle is at a standstill, while " 5 " means the vehicle can travel across 5 cells within one time step and the corresponding speed is $90 \mathrm{~km} / \mathrm{h}$. On the other hand, the maximum speed of any autonomous vehicle is 7 cells per time step (i.e., $126 \mathrm{~km} / \mathrm{h}$ ). A cell is in either of two states at any time instant, vacant or occupied by a vehicle. With the vehicle speed information taken into account, we may view the state of each cell as varying from -1 to 7 , with " -1 " addressing the vacant status and " $0-7$ " the occupying status with the corresponding vehicle speed at level 0-7.

2.2. Basic Rules for Updating System State. Some rules are based to update the states of all vehicles over one time step from $t$ to $t+1$. Each rule addresses a specific maneuver of vehicles. In the sequence of how the system is updated in each time step, these rules are presented as follows:

(i) Lane changing,

(ii) Acceleration: $v_{n} \rightarrow \min \left(v_{\max }, v_{n}+1\right)$,

(iii) Deterministic deceleration: $v_{n} \rightarrow \min \left(v_{n}, d_{n}\right)$,

(iv) Randomization: $v_{n} \rightarrow \max \left(v_{n}-1,0\right)$,

(v) Position updates: $x_{n} \rightarrow x_{n}+v_{n}$, 
where $v_{n}(t)$ denotes the velocity of a certain vehicle $n$ at time instant $t, v_{\max }$ denotes the maximum velocity limit, which is equal to either 5 or 7 in this work, $d_{n}$ represents the front spacing of vehicle $n$ on the same lane, and $x_{n}$ stands for the current position of vehicle $n$.

For every time step, these rules are sequentially considered for each vehicle to update its state, and the lanechanging maneuver is always conducted first, of which the rules are detailed in Section 2.3. The acceleration rule says that any vehicle $n$ may not increase its speed over one time step by more than one level. The deceleration rule means that a vehicle keeps its current speed unless it has to slow down to adapt to its current front spacing. The randomization rule applies only to RVs with the probability $p \in(0,1)$. This means that a percentage of drivers are not able to maintain the current speed constantly and may randomly slow down their vehicles. The position-updating rule states that vehicles update their locations according to their current speeds. This rule is taken from the single-lane NaSch model.

2.3. Lane-Changing Rules. Any vehicle, AV or RV, is assumed to obey a basic rule that lane changing could be conducted only at the least cost of speed reduction of neighboring vehicles; that is, a vehicle would change lane only if its neighboring vehicles in the target lane would not have to accordingly slow down too much. In addition, it is assumed that the left lane changing has the first priority if lane changing in either direction is acceptable.

The major difference between RVs and AVs in terms of lane changing is that an AV could communicate with adjacent AVs in the target lanes for the possibility of more flexible lane changing. More specifically, two sets of lane-changing rules are considered, which are Polite Lane Changing (PLC) and Aggressive Lane Changing (ALC) rules. Depending on the situation, an AV may follow either PLC or ALC while an RV can only follow the ALC rule.

(1) Polite Lane Changing (PLC). The fundamental hypothesis concerning PLC is that drivers are cautious when they attempt to change lanes; that is, their lane-change maneuvers are not supposed to interfere with the motion of neighboring vehicles on the adjacent lanes. This is basically consistent with the MOBIL model with its politeness parameter equal to 1 . Instead of assuming self-centered behavior in most lane-changing models, PLC rules support a more altruistic behavior.

Generally speaking, two aspects need to be taken into account while studying lane changing: the incentive and safety. Only if some criteria concerning both aspects are met will vehicles change lanes. We make reference to the STCA model [19] to set up the incentive and safety criteria for PLC. The PLC rules are illustrated with Figure 1, where vehicles in red represent $\mathrm{AVs}$ s preparing to change lanes.

As shown in Figure 1(a), vehicle $n$ takes the left lane as the target lane and may conduct lane changing if the following conditions are met:

$$
d_{n}<\min \left(v_{n}(t)+1, v_{\max }\right)
$$

$$
\begin{gathered}
d_{n, l}>d_{n} \\
d_{n, l, \text { back }}>v_{\max },
\end{gathered}
$$

where $v_{n}(t)$ denotes the velocity of the $n$th vehicle at time instant $t, v_{\max }$ denotes the maximum velocity limit of the $n$th vehicle, and $d_{n}, d_{n, l}$, and $d_{n, l, \text { back }}$ represent the front spacing of vehicle $n$ on the same lane, the front spacing of vehicle $n$ on the left lane, and rear spacing of vehicle $n$ on the left lane. Similar nomenclature applies to the right lane-changing case.

Equation (1) formulates the incentive criterion while (2) and (3) address the safety criterion. More precisely, (1) means that current front spacing is not sufficient if the current speed is increased by one level; (2) and (3) mean that the left (target) front spacing is adequate for lane changing and the left rear spacing is absolutely safe. If (1)-(3) are not met, vehicle $n$ checks the possibility of lane changing on the right side:

$$
\begin{gathered}
d_{n, r}>d_{n} \\
d_{n, r, \text { back }}>v_{\max } .
\end{gathered}
$$

If (1), (4), and (5) are not met, vehicle $n$ remains in the current lane. When (1)-(3) or (1), (4), and (5) are met, vehicle $n$ could do lane changing with a probability of $P_{c}$. This parameter is introduced to reflect the individuality of vehicles in lane changing (i.e., even under the same conditions that favor lane changing, some vehicles may not choose to do it).

As plotted in Figures 1(b) and 1(c), two more sets of rules are considered to address cooperative lane changing of two AVs:

$$
\begin{aligned}
d_{n+2, r, \text { back }} & >v_{\max } \\
d_{n, l, \text { back }}+l_{n}+d_{n} & \geq v_{n+2}(t) \\
d_{n+2, l, \text { back }} & >v_{\max } \\
d_{n+2, l} & \geq v_{n+2}(t),
\end{aligned}
$$

where $l_{n}$ denotes the length of vehicle $n$. Equations (6) and (7) correspond to Figure 1(b), where the two AVs cooperate via intercommunication to do lane changing in the opposite directions. Equations (8) and (9) address the case of Figure 1(c), where the two AVs cooperate to do lane changing in the same direction. The probability parameter $P_{c}$ applies.

The PLC rules are assumed in this work to be obeyed only by AVs, while the ALC rules to be introduced below can be followed by both AVs and RVs.

(2) Aggressive Lane Changing (ALC). ALC represents a set of more realistic lane-changing rules than PLC, especially for RVs. Research on the lane-changing behavior of RVs [35, 36 indicates that slower preceding vehicles would in many situations tempt the following drivers to consider overtaking. In addition, $95 \%$ of drivers would choose to do lane changing only if the rear spacing on the target lane is bigger than a 3cell length (15 meters) and their speeds are higher than the following vehicles on the target lane [37,38]. As inspired by 


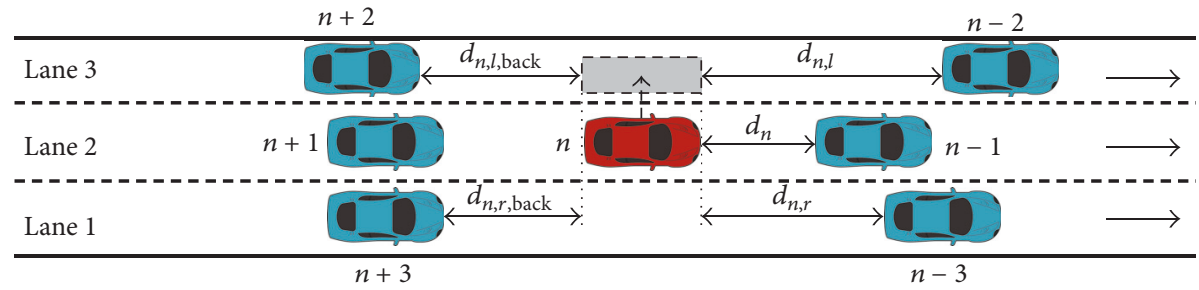

(a) Basic lane changing

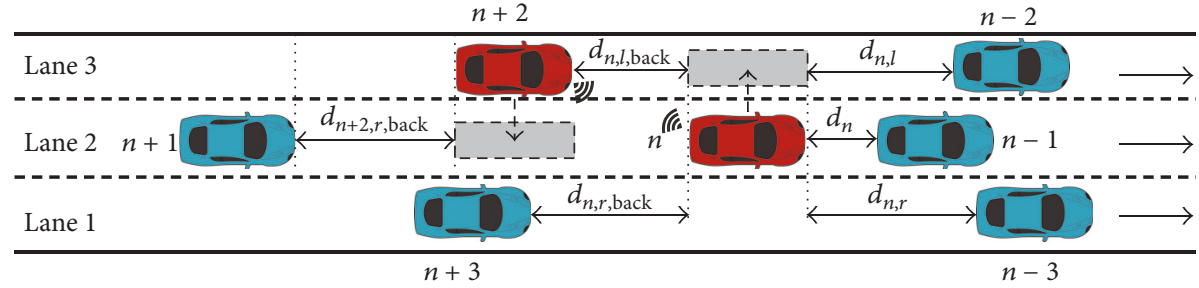

(b) Cooperative lane changing in opposite directions

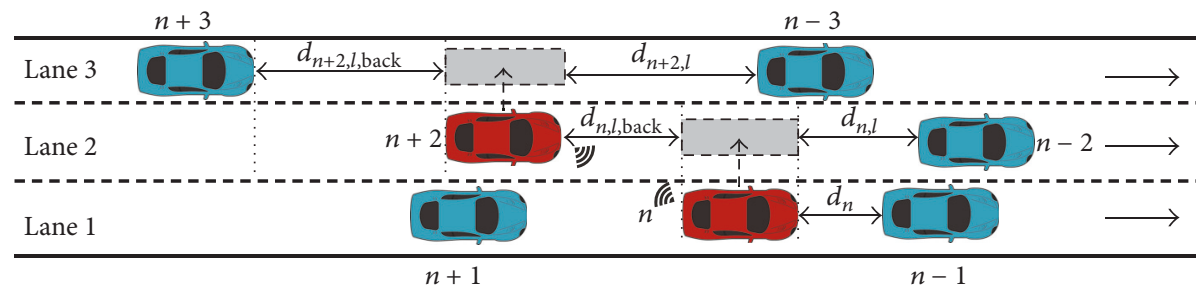

(c) Cooperative lane changing in the same direction

FIgURE 1: The lane-changing rules and simulation scenarios.

these findings, we have designed the ALC rules to supplement the PLC rules:

$$
\begin{aligned}
d_{n, \text { target,back }} & \geq d_{0} \\
v_{n}(t) & \geq v_{n, \text { target,back }}(t) .
\end{aligned}
$$

If vehicle $n$ is an RV, $d_{0}$ is set to be equal to 3-cell length; otherwise, $d_{0}$ is set equal to a 2-cell length to represent the different accuracy of driving behavior of AVs and RVs.

(3) PLC or ALC for an AV. Given an AV at a certain time instant, if the conditions for it to do PLC are met, it chooses PLC; otherwise, if the conditions for ALC are satisfied, it opts for ALC with aggression under a certain probability $p_{\text {aggressive }}$, which indicates that this maneuver will influence adjacent vehicles' velocities. However, if neither lane-changing maneuver is possible, it remains in the current lane (see also Figure 3).

\section{Numerical Simulation and Result Analysis}

3.1. Simulation Setup. As shown in Figure 1, a freeway stretch of 3 lanes without on/off-ramps was considered for the simulation studies. The stretch consists of 50 cells (i.e., the stretch length is $250 \mathrm{~m}$ ). The maximum speed of a regular or autonomous vehicle is 5 or 7 cells per time step. Let $N$, $N_{a}$, and $N_{r}$ denote the total number of vehicles, AVs, and RVs within the simulated stretch. As routine practice in the cellular automata based simulations, the periodic boundary condition is considered. At the start of each simulation, only some cells are occupied with vehicles and these initial locations are randomly selected.

Denote by $Q, \bar{v}, \rho$, and $\rho_{i}$ the average flow, the average speed, the average density of the stretch, and the density of the lane $i(i=1,2,3)$. More specifically,

$$
\begin{aligned}
\bar{v} & =\frac{1}{T} \sum_{t=t_{0}}^{T+t_{0}-1} \frac{1}{N} \sum_{n=1}^{N} v_{n}(t) \\
\rho & =\frac{N}{3 L} \\
\rho_{i} & =\frac{N_{i}}{L}
\end{aligned}
$$

and the flow can be determined as

$$
Q=\rho \cdot \bar{v} .
$$

The simulation was run 20 times for each scenario. The simulation time horizon $T$ is 20,000 time steps, and for each simulation run, the outcome from the first $10^{4}$ simulation time steps was discarded in order to remove transient effects, and the results of the next $10^{4}$ time steps were recorded.

3.2. The Impact of AVs on Traffic Flow Characteristics. Figure 2 shows the characteristics of mixed traffic flow, 


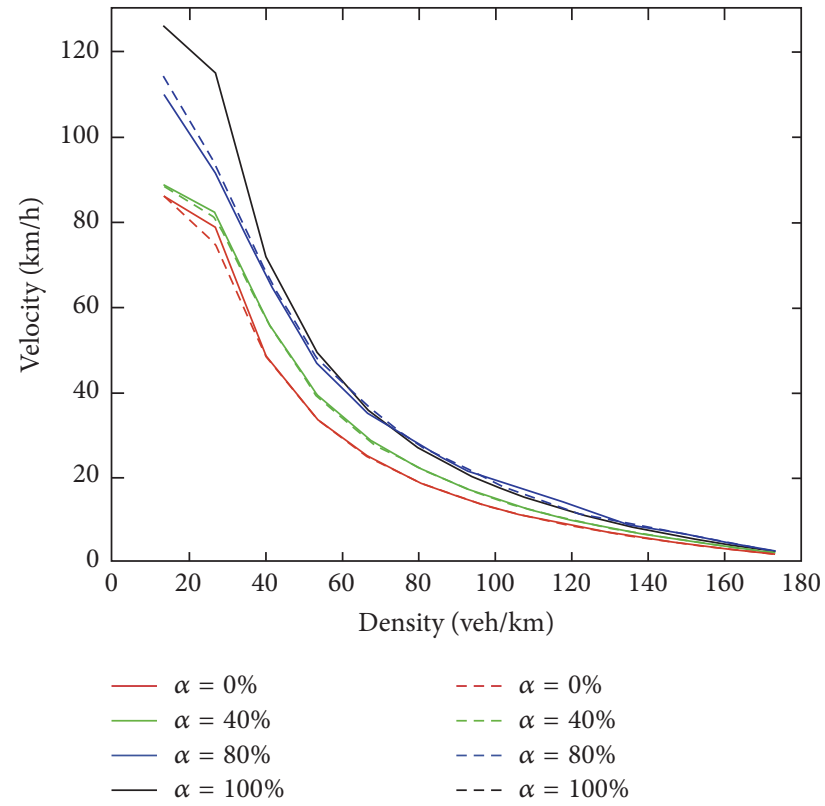

(a)

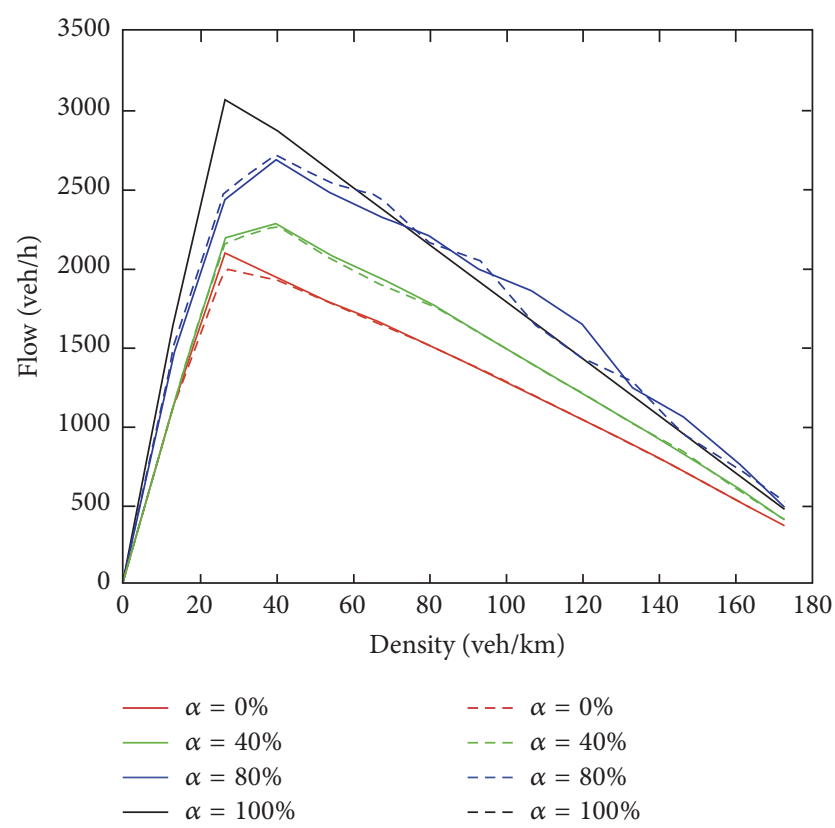

(b)

FIGURE 2: Fundamental diagrams of mixed traffic flow.

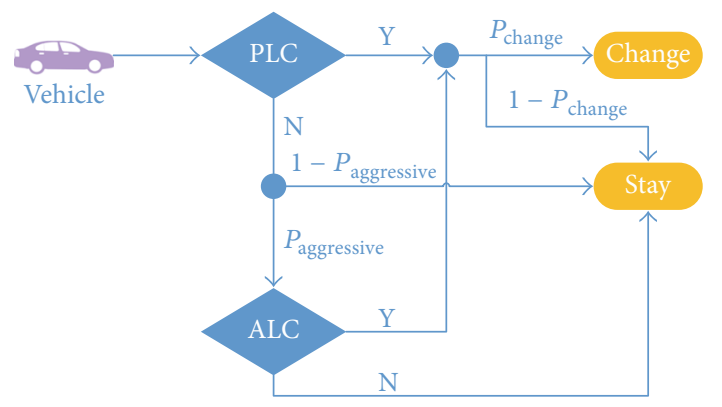

Figure 3: PLC or ALC for an AV.

where the legend addresses different $\mathrm{AV}$ proportions (i.e., the market penetration rate of $\mathrm{AVs}$ ), and the solid/dash line for each AV proportion refers to the PLC/ALC rules. First, Figure 2(a) presents the speed-density relation obtained with the simulation data. With the increase of the AV proportion, the speed-density curve moves to the right. This indicates that, first, the increase of the AV ratio increases the mean speed given the same density, and second, with more AVs involved, the entire traffic flow could endure a higher density for any given speed. All these are basically consistent with our general understanding of the capacity of AVs, particularly their negligible reaction time delays. Second, Figure 2(b) presents the flow-density relation extracted from the simulation data. Clearly, the inclusion of AVs considerably increases the road capacity and free-flow speed. More specifically, the road capacity is about $2000 \mathrm{vehs} / \mathrm{h}$ at zero percentage of AVs $(\alpha=0)$ and becomes 3070 vehs $/ \mathrm{h}$ when all vehicles are AVs $(\alpha=100 \%)$. The free-flow speed is about $78.85 \mathrm{~km} / \mathrm{h}$ when $\alpha=0$ and becomes $115.20 \mathrm{~km} / \mathrm{h}$ when $\alpha=100 \%$, about
46\% higher. In addition, the increase of the AV proportion also improves the critical density slightly, indicating that the introduction of AVs makes the entire traffic flow more stable. On the other hand, the use of different rules of lane changing (PLC or ALC) seems to have made little difference. Further studies are needed for a more concrete conclusion on this issue.

3.3. The Impact of Lane-Changing Probability. As mentioned, when some preset conditions are met, vehicles may change lanes or not, and in our simulation studies, a probability $P_{c}$ is assigned so that only a portion of candidate vehicles choose to change lanes. Figure 4 illustrates the impact of $P_{c}$ on the fundamental diagram under different $\mathrm{AV}$ proportions, where only the ALC lane-changing rule is considered. Clearly, there is a contrast between Figures 2 and 4: the involvement of AVs does improve traffic flow characteristics a lot (Figure 2) while the impact of lane changing on flow seems to be much less than expected. This may indicate that the change in the carfollowing maneuvers of AVs (compared to RVs) contributes more to the improvement of traffic flow characteristics than the smarter lane-changing operation of AVs. To further investigate the impact of lane changing, next we look into the lane-wise lane-changing frequency for details.

3.4. Lane-Changing Frequency. In this section, we focus on the case of $P_{c}=1$ and explore the details of lane-wise lane changing. First of all, the lane-changing frequency $f_{i, j}$ between lanes $i$ and $j$ is defined as follows:

$$
f_{i, j}=\frac{N_{i, j \text { llane-changing }}}{T \cdot N}
$$



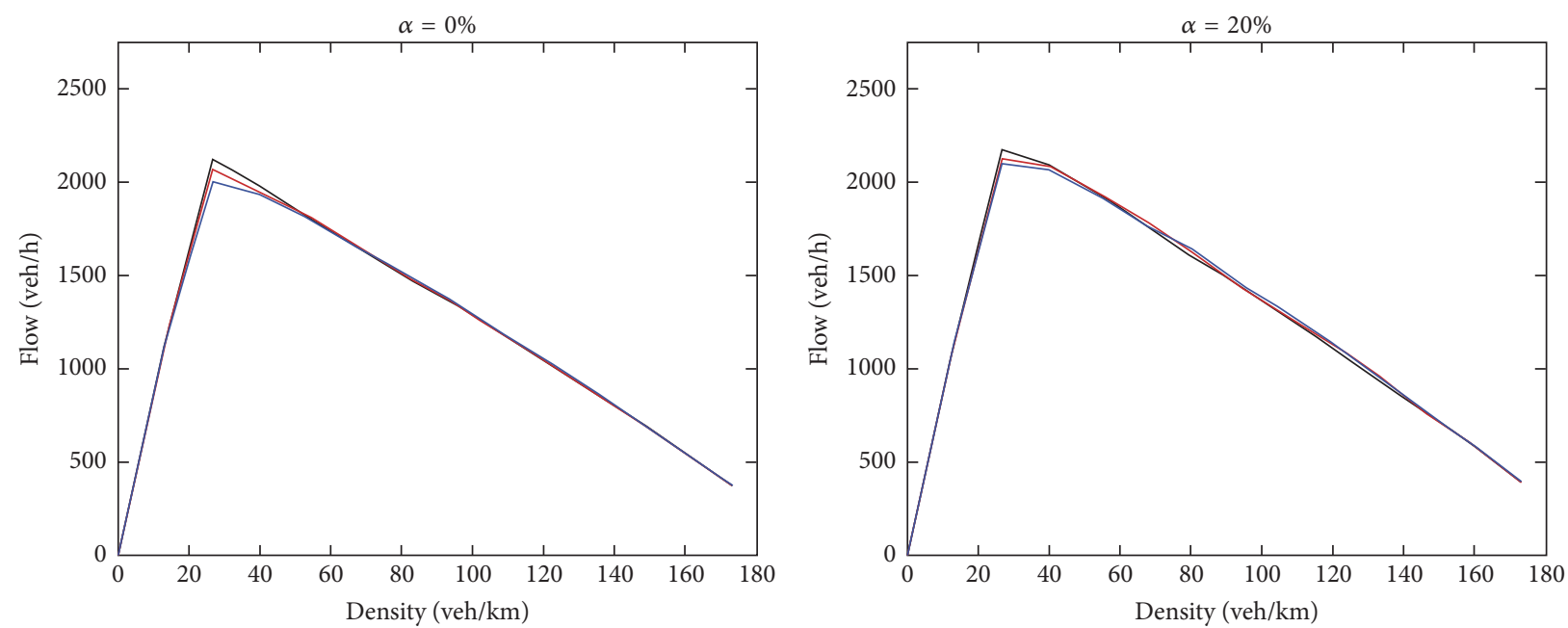

- $P_{c}=0.4$

$-P_{c}=0.8$

$-P_{c}=1.0$



$-P_{c}=0.4$

$-P_{c}=0.8$

$-P_{c}=1.0$



$$
\begin{aligned}
& -P_{c}=0.4 \\
& -P_{c}=0.8 \\
& -P_{c}=1.0
\end{aligned}
$$
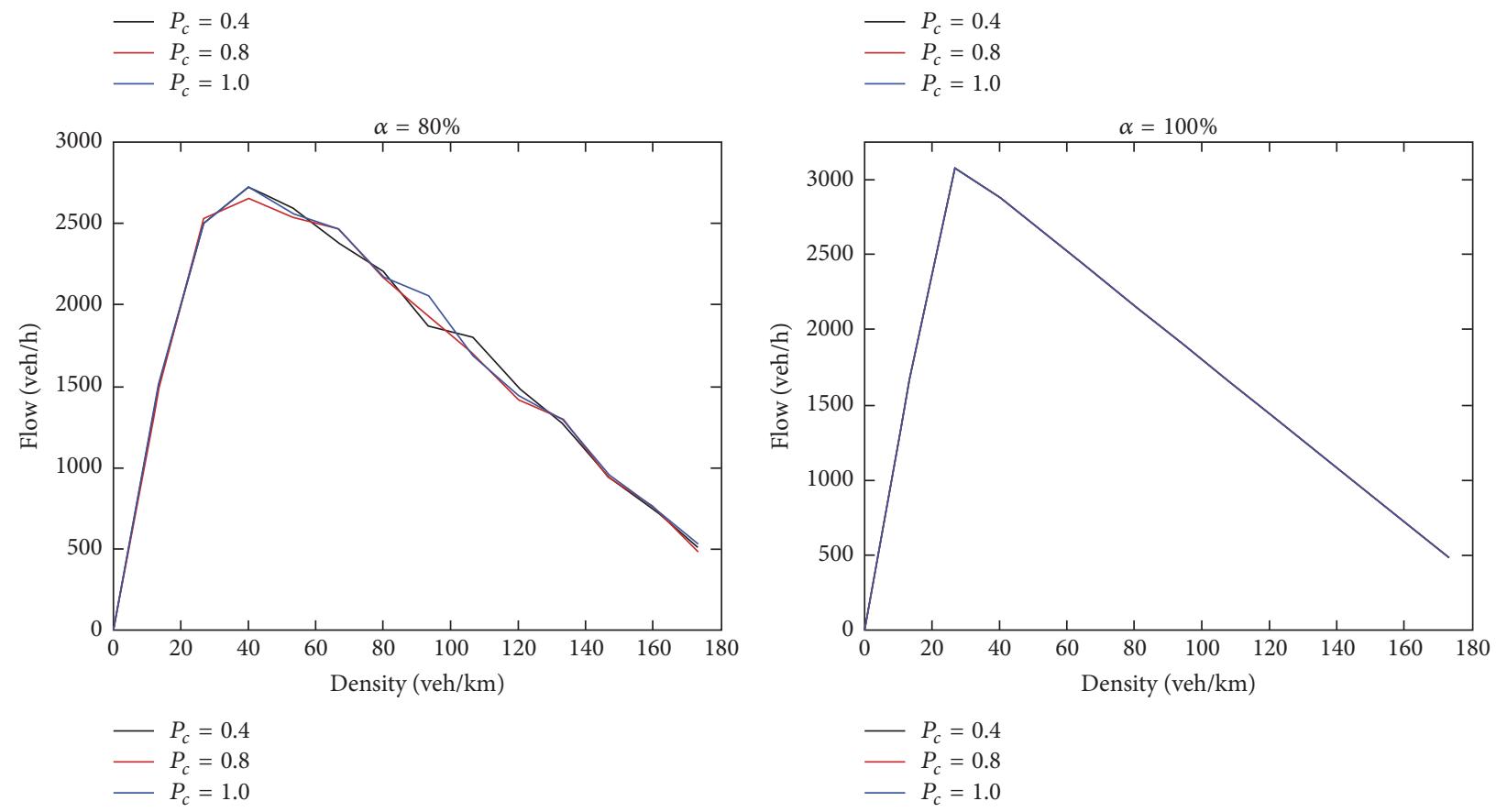$$
\begin{aligned}
-P_{c} & =0.4 \\
-P_{c} & =0.8 \\
-P_{c} & =1.0
\end{aligned}
$$

FIgURE 4: Fundamental diagrams for different lane-changing probabilities $\left(P_{c}\right)$. 


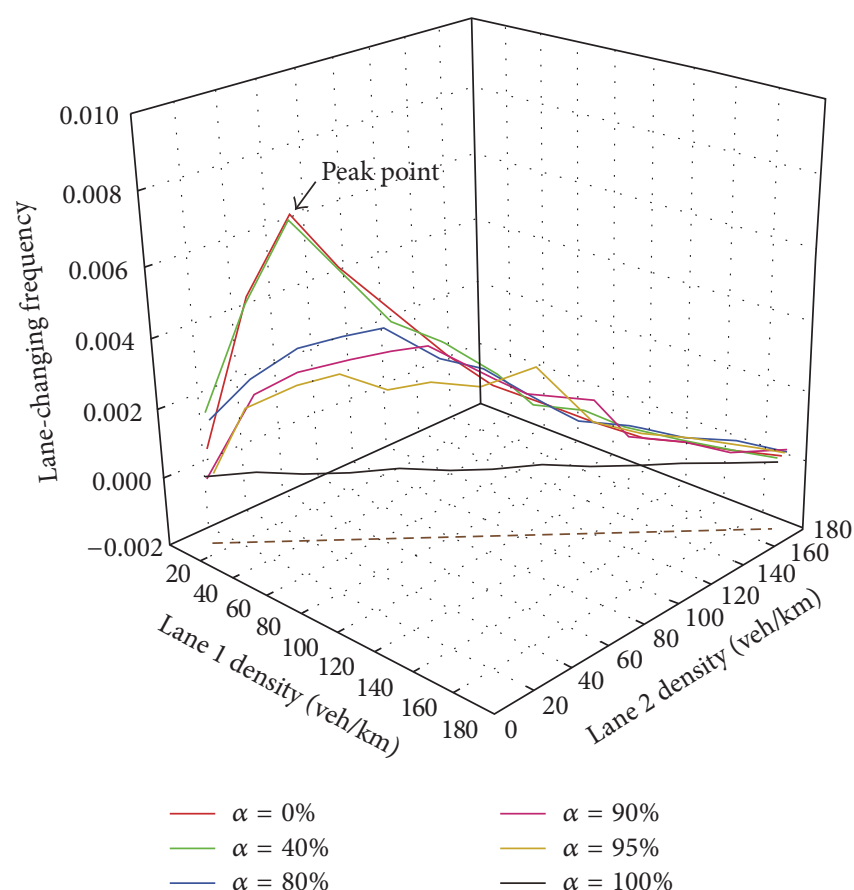

(a)

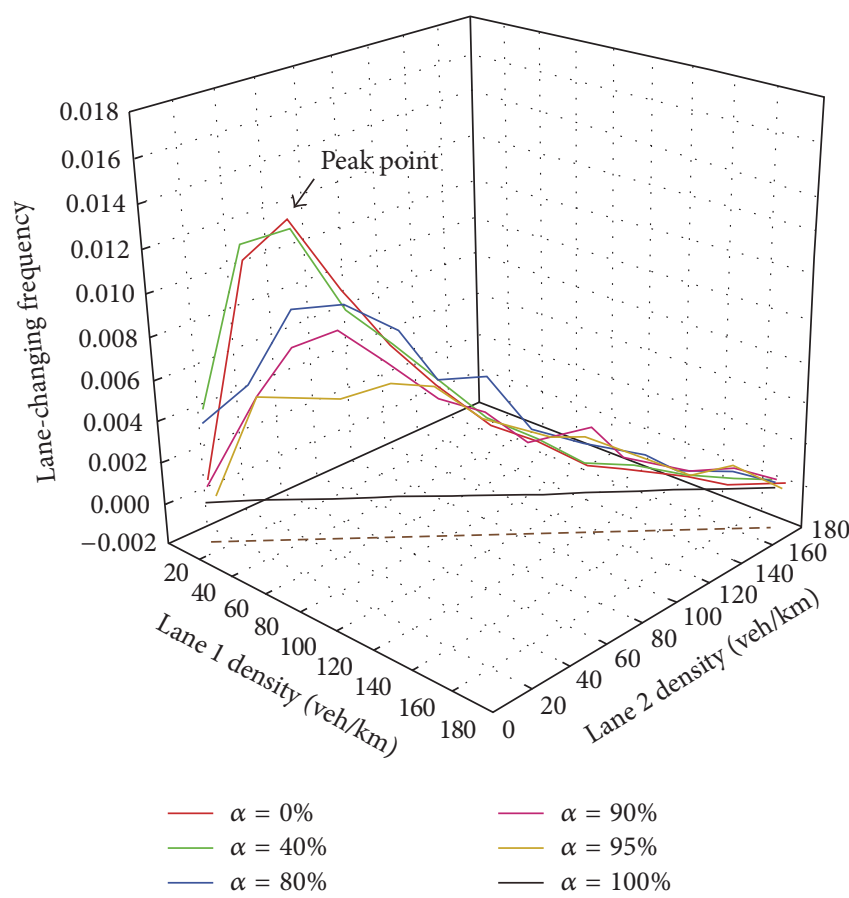

(c)

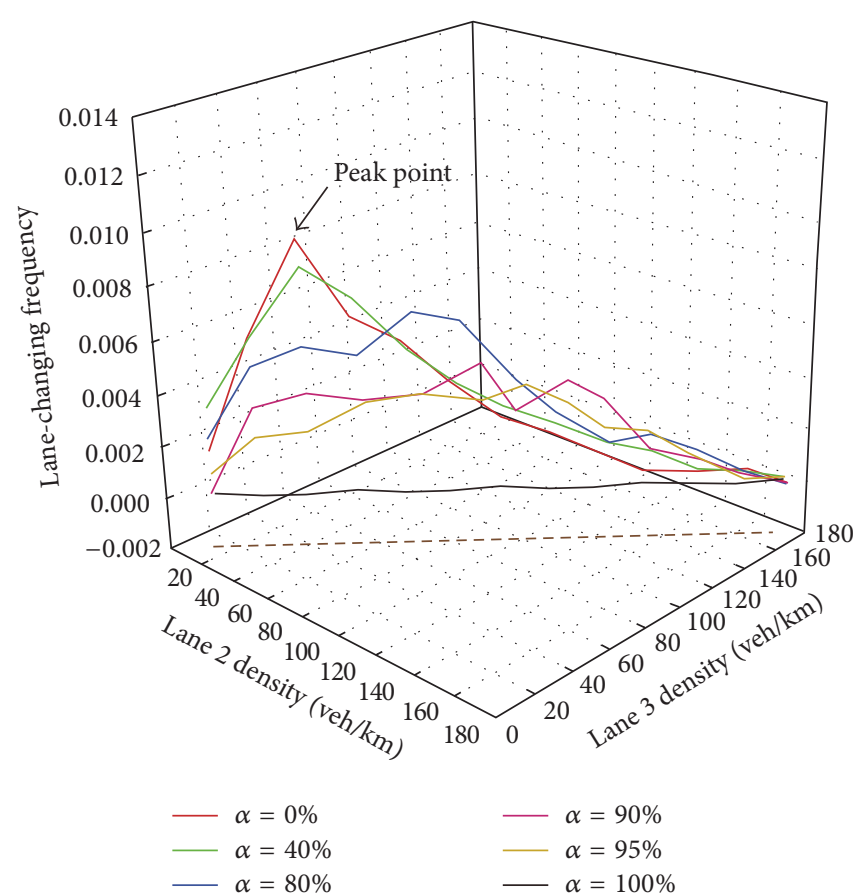

(b)

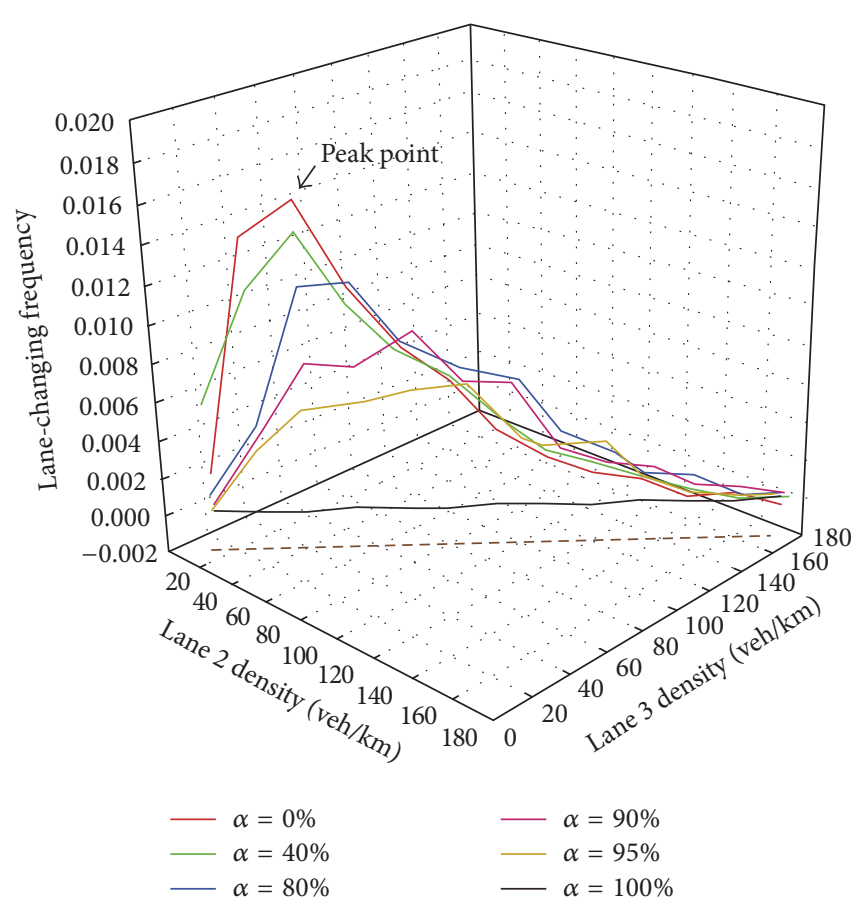

(d)

FIgURe 5: Lane-changing frequency of mixed traffic flow: (a, c) $i=1$ and $j=2$; (b, d) $i=2$ and $j=3$; (a, b) PLC; (c, d) ALC.

where the numerator refers to the total number of lanechanging instances between lane $i$ and lane $j$ over the simulation horizon $T$, while the denominator represents the total number of vehicles involved in the entire simulation.

The simulation results are presented in Figure 5, where the $z$ (height) dimension of each subfigure refers to $f_{i, j}(\%)$ and the $x$ and $y$ dimensions refer to traffic densities of lanes $i$ and $j$. Figures 5(a) and 5(c) address the case where $i=1$ and $j=2$, and Figures 5(b) and 5(d) address the case where $i=2$ and $j=3$. Moreover, Figures 5(a) and 5(b) are with the PLC rule, and Figures 5(c) and 5(d) are with the ALC rule. For simplicity, the values of densities are taken along the dashed diagonal line on the bottom plane in each subfigure.

Focusing on Figure 5(a), we see that, with each AV ratio alpha, $f_{i, j}$ evolves along a fundamental-diagram-like curve over densities of lanes $i$ and $j$, and this is in fact consistent 
TABLE 1: Turning points in the fundamental-diagram-like curves of $f_{i, j}$ over densities.

\begin{tabular}{lccccc}
\hline \multirow{2}{*}{ Scenario } & \multicolumn{3}{c}{ Lane density $[\mathrm{veh} / \mathrm{km}]$} & $\alpha$ & Lane-changing frequency \\
& $\rho_{1}$ & $\rho_{2}$ & $\rho_{3}$ & & \\
\hline (a) & 38.7 & 41.02 & & $0 \%$ & 0.0723 \\
(b) & & 41.02 & 40.29 & $0 \%$ & 0.0096 \\
(c) & 38.12 & 40.76 & & $0 \%$ & 0.0131 \\
(d) & & 40.76 & 41.12 & $0 \%$ & 0.0160 \\
\hline
\end{tabular}

with our intuitive understanding; that is, when the densities of both lanes are low, vehicles have more freedom to do lane changing, and $f_{i, j}$ increases with the number of vehicles on roads until reaching a critical/turning point and then starts to decrease. In addition, the more the AVs involved, the less the value of $f_{i, j}$. This is because AVs can manage the car-following process more efficiently even with much smaller intervehicle spacing than RVs and hence have less incentive to change lanes. And in the extreme case when all vehicles are AVs, there is no need at all to do any lane changing as shown with the black curve in Figure 5(a). Comparison of Figures 5(a) and 5(c) with Figures 5(b) and 5(d) shows that using ALC rather than PLC can substantially increase $f_{i, j}$, though the impact on the overall traffic flow characteristics is not so obvious (see solid and dashed lines in Figure 2).

It is interesting to mention that the turning points in Figure 5 are in most cases quite close to those in Figure 2 in terms of traffic densities (around 40 vehs $/ \mathrm{km}$ ) (see Table 1 for details). This indicates that when the underlying traffic densities are low, more lane changes are welcome and also beneficial for better usage of road capacity. And around the critical point, both the incentive and the possibility of lane changing are maximized while the flow also reaches its peak. After that, lane changing becomes less and less practical. The simulation results also indicate that the critical/turning points are not much sensitive to the $\mathrm{AV}$ ratio values.

3.5. Congestion Degree. Whenever a vehicle's speed is at the level of 0 or 1 cell per time step, we say that the vehicle is experiencing congestion, and thus the congestion degree (CD) is calculated as follows:

$$
\mathrm{CD}=\frac{N_{c}}{T \cdot N}
$$

where $N_{c}$ denotes the number of congested vehicles over the whole simulation horizon $T$ and $T \cdot N$ denotes the total number of vehicles involved in the whole simulation horizon. It is natural that CD increases with density (see Figure 6). The presence of AVs does lower CD under the same density conditions. In particular, before density reaches the critical density of $40 \mathrm{vehs} / \mathrm{km}$, which corresponds to the capacity flow, the involvement of AVs definitely helps to keep CD at a lower level. Throughout the whole density spectrum, it seems that the mixed traffic flow with an AV proportion of $50 \%$ works best to maintain a constantly lower CD. The authors are doing further work to explore the mechanism underpinning this observation.

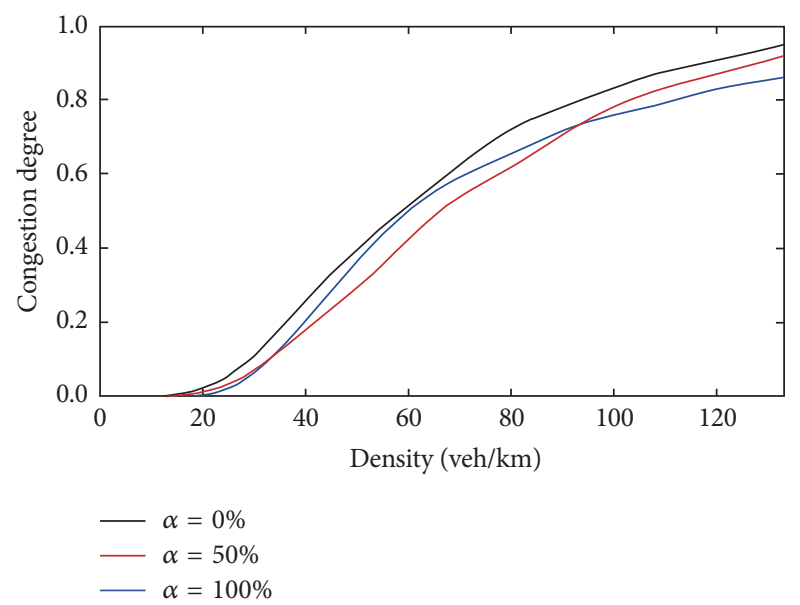

FIGURE 6: Congestion degree.

\section{Conclusions}

This paper has studied the impact of autonomous vehicles on the characteristics of mixed traffic flow. As no autonomous vehicle is really running on roads, current investigations are mainly performed via simulation. Based on an improved cellular automata model, a simulation platform was developed for this work.

The following conclusions are obtained through intensive simulation studies:

(1) Traffic capacity and free-flow speed increase positively with the penetration rate of autonomous vehicles.

(2) The lane-changing frequency between neighboring lanes evolves with traffic density along a fundamentaldiagram-like curve.

(3) The impact on the overall traffic flow characteristics of smart lane changing by autonomous vehicles seems to be much less pronounced than that of smart car following.

Further work is being conducted to address extended and more practical traffic scenarios.

\section{Conflicts of Interest}

The authors declare that there are no conflicts of interest.

\section{Authors' Contributions}

Yangzexi Liu and Jingqiu Guo contributed equally to this study.

\section{Acknowledgments}

This work was supported in part by the Zhejiang Qianren Program of China 2013-2018, Shanghai Pujiang Program, and the National Natural Science Foundation of China under Grants 51478428 and 71671126. 


\section{References}

[1] Y.-M. Yuan, R. Jiang, M.-B. Hu, Q.-S. Wu, and R. Wang, "Traffic flow characteristics in a mixed traffic system consisting of ACC vehicles and manual vehicles: A hybrid modelling approach," Physica A: Statistical Mechanics and its Applications, vol. 388, no. 12, pp. 2483-2491, 2009.

[2] M. P. Enoch, "How a rapid modal convergence into a universal automated taxi service could be the future for local passenger transport," Technology Analysis and Strategic Management, vol. 27, no. 8, pp. 910-924, 2015.

[3] J. Wang, C. Wang, J. Lv, Z. Zhang, and C. Li, "Modeling Travel Time Reliability of Road Network Considering Connected Vehicle Guidance Characteristics Indexes," Journal of Advanced Transportation, vol. 2017, pp. 1-9, 2017.

[4] C. Roncoli, I. Papamichail, and M. Papageorgiou, "Model predictive control for motorway traffic with mixed manual and VACS-equipped vehicles," pp. 452-461.

[5] Separation of Vehicles-CMV-Only Lanes, National Academies Press, Washington, D.C., 2010.

[6] J. Ma, X. Li, F. Zhou, J. Hu, and B. B. Park, "Parsimonious shooting heuristic for trajectory design of connected automated traffic part II: computational issues and optimization," Transportation Research Part B: Methodological, vol. 95, pp. 421-441, 2017.

[7] F. Zhou, X. Li, and J. Ma, "Parsimonious shooting heuristic for trajectory design of connected automated traffic part I: theoretical analysis with generalized time geography," Transportation Research Part B: Methodological, vol. 95, pp. 394-420, 2017.

[8] M. W. Levin and S. D. Boyles, "A multiclass cell transmission model for shared human and autonomous vehicle roads," Transportation Research Part C: Emerging Technologies, vol. 62, pp. 103-116, 2016.

[9] U. Khan, P. Basaras, L. Schmidt-Thieme, A. Nanopoulos, and D. Katsaros, "Analyzing cooperative lane change models for connected vehicles," in Proceedings of the 3rd International Conference on Connected Vehicles and Expo (ICCVE '14), pp. 565-570, November 2014.

[10] S. E. Shladover, C. Nowakowski, X.-Y. Lu, and R. Ferlis, “Cooperative adaptive cruise control: Definitions and operating concepts," Transportation Research Record, vol. 2489, pp. 145-152, 2015.

[11] L. Du, L. Han, and S. Chen, "Coordinated online in-vehicle routing balancing user optimality and system optimality through information perturbation," Transportation Research Part B: Methodological, vol. 79, pp. 121-133, 2015.

[12] X. Qu, S. Wang, and J. Zhang, "On the fundamental diagram for freeway traffic: a novel calibration approach for single-regime models," Transportation Research Part B: Methodological, vol. 73, pp. 91-102, 2015.

[13] M. Zhou, X. Qu, and S. Jin, "On the impact of cooperative autonomous vehicles in improving freeway merging: a modified intelligent driver model-based approach," IEEE Transactions on Intelligent Transportation Systems, vol. PP, no. 99, pp. 1-7, 2016.

[14] X. Qu, J. Zhang, and S. Wang, "On the stochastic fundamental diagram for freeway traffic: Model development, analytical properties, validation, and extensive applications," Transportation Research Part B: Methodological, vol. 104, pp. 256-271, 2017.

[15] K. Nagel and M. Schreckenberg, "A cellular automaton model for freeway traffic," Journal de Physique I, vol. 2, no. 12, pp. 22212229, 1992.
[16] A. Fotheringham and P. Rogerson, The SAGE Handbook of Spatial Analysis, SAGE Publications, Ltd, 1 Oliver's Yard, 55 City Road, London England EC1Y 1SP United Kingdom , 2009.

[17] S. Openshaw and R. J. Abrahart, GeoComputation, Taylor \& Francis, Abingdon, UK, 2000.

[18] S. Maerivoet and B. De Moor, "Cellular automata models of road traffic," Physics Reports. A Review Section of Physics Letters, vol. 419, no. 1, pp. 1-64, 2005.

[19] D. Chowdhury, D. E. Wolf, and M. Schreckenberg, "Particle hopping models for two-lane traffic with two kinds of vehicles: effects of lane-changing rules," Physica A: Statistical Mechanics and Its Applications, vol. 235, no. 3-4, pp. 417-439, 1997.

[20] M. M. Pedersen and P. T. Ruhoff, "Entry ramps in the NagelSchreckenberg model," Physical Review E, vol. 65, no. 2, Article ID 056705, 9 pages, 2002.

[21] S. Jin, X. Qu, C. Xu, D. Ma, and D. Wang, "An improved multivalue cellular automata model for heterogeneous bicycle traffic flow," Physics Letters, Section A: General, Atomic and Solid State Physics, vol. 379, no. 39, Article ID 23345, pp. 2409-2416, 2015.

[22] Y. J. Luo, B. Jia, J. Liu, W. H. K. Lam, X. G. Li, and Z. Y. Gao, "Modeling the interactions between car and bicycle in heterogeneous traffic," Journal of Advanced Transportation, vol. 49, no. 1, pp. 29-47, 2015.

[23] N. Moussa and A. K. Daoudia, "Numerical study of two classes of cellular automaton models for traffic flow on a two-lane roadway," European Physical Journal B, vol. 31, no. 3, pp. 413420, 2003.

[24] X.-G. Li, B. Jia, Z.-Y. Gao, and R. Jiang, "A realistic two-lane cellular automata traffic model considering aggressive lanechanging behavior of fast vehicle," Physica A: Statistical Mechanics and its Applications, vol. 367, pp. 479-486, 2006.

[25] P. Hidas, "Modelling vehicle interactions in microscopic simulation of merging and weaving," Transportation Research Part C: Emerging Technologies, vol. 13, no. 1, pp. 37-62, 2005.

[26] Z. Zheng, S. Ahn, D. Chen, and J. Laval, "Freeway traffic oscillations: Microscopic analysis of formations and propagations using Wavelet Transform," Transportation Research Part B: Methodological, vol. 45, no. 9, pp. 1378-1388, 2011.

[27] J. C. McCall, D. P. Wipf, M. M. Trivedi, and B. D. Rao, "Lane change intent analysis using robust operators and sparse Bayesian learning," IEEE Transactions on Intelligent Transportation Systems, vol. 8, no. 3, pp. 431-440, 2007.

[28] P. G. Gipps, "A model for the structure of lane-changing decisions," Transportation Research Part B: Methodological, vol. 20, no. 5, pp. 403-414, 1986.

[29] Q. Yang and H. N. Koutsopoulos, "A microscopic traffic simulator for evaluation of dynamic traffic management systems," Transportation Research Part C: Emerging Technologies, vol. 4, no. 3, pp. 113-129, 1996.

[30] K. I. Ahmed, M. E. Ben-Akiva, H. N. Koutsopoulos, and R.G. Mishalani, "Models of freeway lane changing and gap acceptance behavior," Proceedings of the 13th International Symposium on Transportation and Traffic Theory, Lyon, France, 501-515, 1996.

[31] O. A. Osman, S. Ishak, and M. Ye, "Crash Risk Aanlysis of Distracted Driving Behavior: Influence of Secondary Task Engagement and Driver Characteristics," in Proceedings of the 5th Annual International Conference on Architecture and Civil Engineering (ACE 2017), pp. 42-45. 
[32] A. Kesting, M. Treiber, and D. Helbing, "General lanechanging model MOBIL for car-following models," Transportation Research Record: Journal of the Transportation Research Board, vol. 1999, no. 1, pp. 86-94, 2007.

[33] Y. Du, Y. Wang, and C.-Y. Chan, "Autonomous lane-change controller via mixed logical dynamical," in Proceedings of the 2014 17th IEEE International Conference on Intelligent Transportation Systems, ITSC 2014, pp. 1154-1159, October 2014.

[34] Z. Zheng, "Recent developments and research needs in modeling lane changing," Transportation Research Part B: Methodological, vol. 60, pp. 16-32, 2014.

[35] S.E Lee, E.C.B. Olsen, and W.W. Wierwille, "A comprehensive examination of naturalistic lane-changes." Publication Dot Hs Virginia Tech Transportation Institute, 2004.

[36] T. G. Oketch, "New modeling approach for mixed-traffic streams with nonmotorized vehicles," Transportation Research Record, no. 1705, pp. 61-69, 2000.

[37] S.-W. Kim and W. Liu, "Cooperative Autonomous Driving: A Mirror Neuron Inspired Intention Awareness and Cooperative Perception Approach," IEEE Intelligent Transportation Systems Magazine, vol. 8, no. 3, pp. 23-32, 2016.

[38] L. Fei, H. B. Zhu, and X. L. Han, "Analysis of traffic congestion induced by the work zone," Physica A: Statistical Mechanics and its Applications, vol. 450, pp. 497-505, 2016. 




\section{Enfincering}


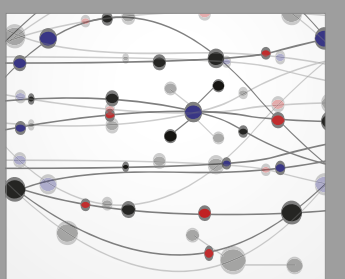

The Scientific World Journal

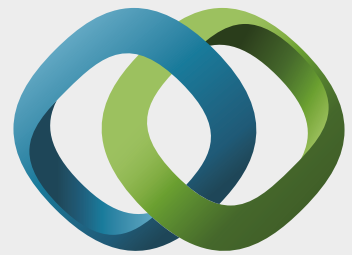

\section{Hindawi}

Submit your manuscripts at

https://www.hindawi.com
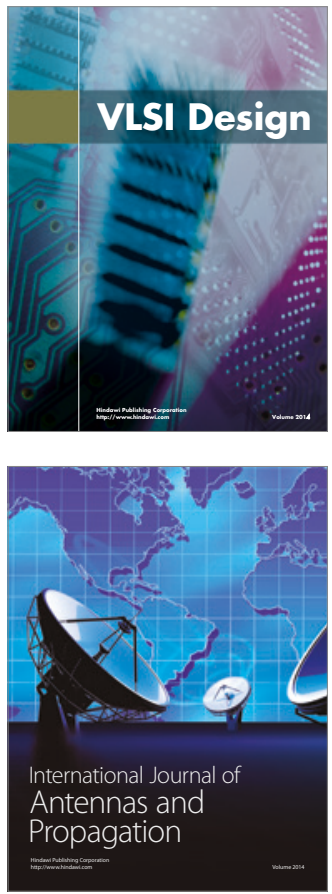

\section{Rotating}

Machinery
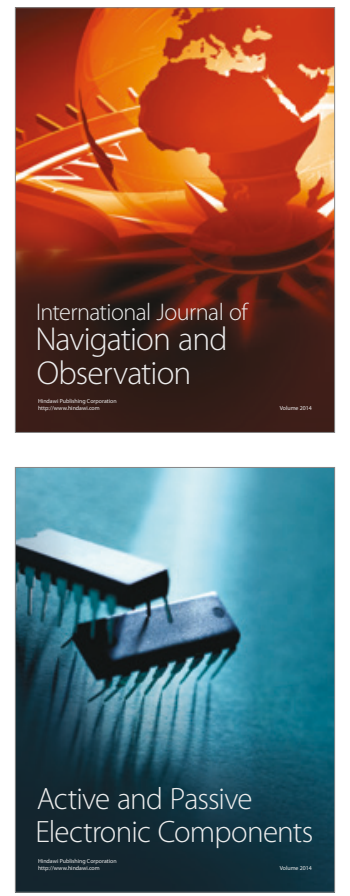
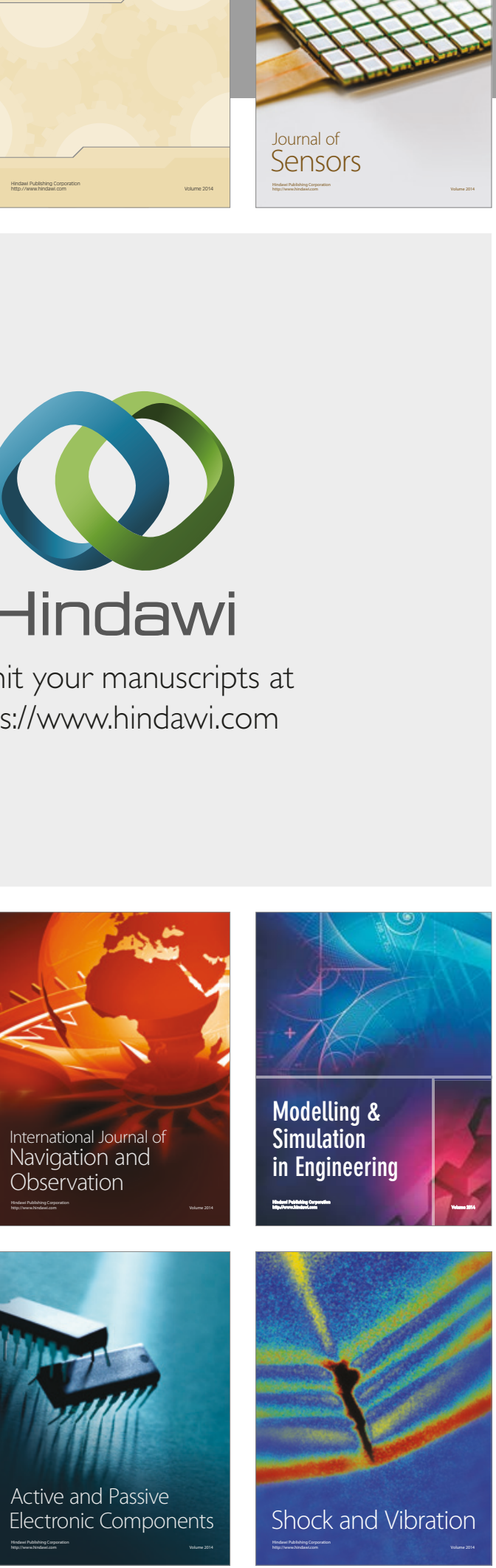
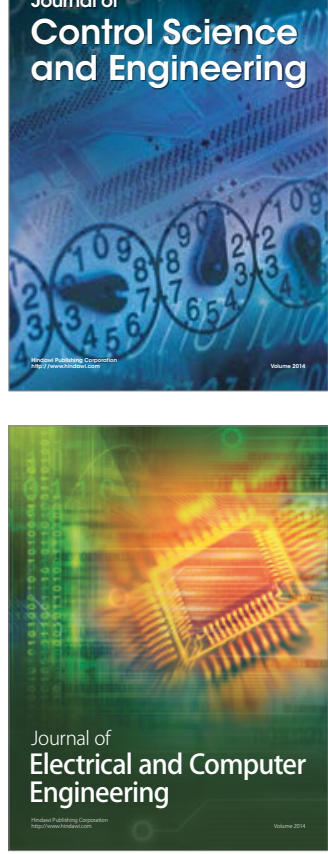

Distributed

Journal of

Control Science

and Engineering
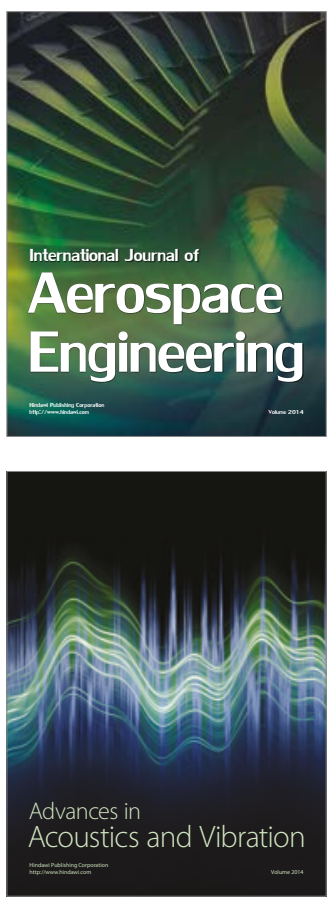

Sensor Networks 